\title{
Tecnologías de información y comunicación en mecanismos de participación ciudadana en el ámbito local ${ }^{*}$
}

\author{
Peña, Dionnys** \\ Olivar, Cira ${ }^{\star \star \star}$ \\ Primera, Nelly ${ }^{\star * \star *}$
}

Resumen

Las tecnologías de información y comunicación (TIC), abren nuevas y efectivas posibilidades para estrechar los lazos gobierno-ciudadanos siempre que estas sean empleadas estratégicamente y aprovechadas al máximo, de éste supuesto surge el objetivo de analizarel aprovechamiento de las TIC en mecanismos de participación ciudadana en elámbito local. Para tal fin se realizó una revisión teórica sobre las opciones tecnológicas que facilitan la interacción, suministran información pública y sean factibles de aplicar en contextos locales, con el propósito de ofrecer lineamientos generales que orienten su incorporación en el funcionamiento de mecanismos de participación implantados en Venezuela. Los resultados revelan que: las tecnologías aptas para apoyar la participación ciudadana son: unidades de información automatizadas, redes basadas en tecnologías de información y gobierno electrónico las cuales pueden constituirse en circuitos de información municipal, donde la capacitación tecnológica figura como un medio para garantizar un mayor y mejor acceso a las mismas por parte de la gestión local y de los ciudadanos. Se concluye que las TIC serán aprovechadas en mecanismos de participación ciudadana en la medida que se logre el compromiso de los actores sociales involucrados para aplicar los lineamientos y estrategias correspondientes.

Palabras clave: Tecnologías de información y comunicación, mecanismos de participación ciudadana, gestión municipal.

Recibido: 17-11-06. Aceptado: 10-10-07

* Este artículo es un avance del proyecto: Tecnologías de información que facilitan la participación ciudadana en la gestión pública local, registrado en el Consejo de Desarrollo Humanístico y Científico (CONDES-LUZ).

** Magíster en Ciencias de la Comunicación. Profesor Agregado de la Universidad del Zulia. Investigador del Centro de Estudios de la Empresa. Maracaibo-Venezuela.

E-mail: dionnysp@gmail.com

*** Lic. Administración. Asistente de investigación adscrita al Centro de Estudios de la Empresa. Cursante de la Maestría en Gerencia Pública.E-mail: cira_elena@yahoo.es

**** Doctora en Ciencias Gerenciales. Profesora Titular de La Universidad del Zulia (LUZ), Investigadora del Centro de Estudios de la Empresa. Maracaibo-Venezuela.

E-mail: nprimera58@hotmail.com 


\title{
Information and Communication Technologies in Citizen Participation Mechanisms in the Local Sphere
}

\begin{abstract}
Information and communication technologies (ICTs), open new and effective possibilities for bringing government and citizens closer together whenever they are used strategically and to the best advantage. Based on this supposition, this study intends to analyze to what degree good use is made of ICTs in citizen participation mechanisms in the local sphere. To accomplish this, a theoretical review was made about technological options that facilitate interaction, supply public information and are feasible to apply in local contexts, with the purpose of offering general guidelines for their incorporation in the functioning of participation mechanisms implanted in Venezuela. Results reveal that the technologies appropriate for supporting citizen participation are automatic information units, networks based on information technologies and electronic government, which can become municipal information circuits, where technological training figures as a means for guaranteeing a greater and better access to the same on by local management and the citizens. Conclusions are that ICTs will be best used in citizen participation mechanisms to the degree that the social actors involved are committed to applying the corresponding guidelines and strategies.
\end{abstract}

Key words: Information and communication technologies, citizen participation mechanisms, municipal management.

\section{Introducción}

La participación ciudadana en los asuntos públicos encuentra en el ámbito local un espacio idóneo para su materialización, ya que éste constituye el nivel de gobierno más cercano a las necesidades y demandas de la comunidad y por ende el que puede darle respuestas rápidas y oportunas a las mismas. Lograr una participación exitosa exige a su vez, sanas relaciones de comunicación entre las instancias del gobierno municipal y los ciudadanos, es aquí donde las tecnologías de información y comunicación (TIC) abren nuevas posibilidades de estrechar estos lazos y permitir que los asuntos de gestión pública sean manejados de manera consensuada, compartida y plural.

Este planteamiento, aunado a las disposiciones constitucionales vigentes en Venezuela, vinculadas con la instauración de mecanismos de participación, llámense Consejos Locales de Planificación Pública (CLPP) o Consejos Comunales (CC) han direccionamiento acciones municipales dedicadas a la búsqueda de salidas para adecuar las TIC a las necesidades de expresión y participación de la sociedad, aprovechando sus bondades en lo que a prestación de servicios públicos e impulso de los principios de transparencia, honestidad y control social se refiere.

Dichas bondades convierten a las TIC en un recurso de gran valía para las autoridades municipales interesadas en el funcionamiento de los mecanismos de participación y algo semejante ocurre con los ciudadanos, quienes una vez en condiciones de usarlas adecuadamente en acciones participativas, podrán benefi- 
Tecnologías de información en mecanismos de participación ciudadana

Peña, Dionnys; Olivar, Cira y Primera, Nelly

ciarse de las potencialidades que éstas ofrecen para tal fin.

En este sentido, los gobiernos municipales han realizados esfuerzos significativos para el aprovechamiento de las TIC, sin embargo, aún persisten problemas caracterizados por la falta de concreción en la selección y aplicación de opciones tecnológicas orientadas al uso de los ciudadanos, pocas iniciativas para estructurar unidades y servicios de información automatizada, dificultades para el acceso a la información pública mediante el empleo de tecnologías, según las investigaciones realizadas por el Programa de las Naciones Unidas para el DesarroIlo-PNUD (2002), Berrizbeitia (2003), Savater citado por Mazzina (2003), Páez et al. (2004) y De Gregorio et al. (2005).

Del mismo modo, estas investigaciones expresan las debilidades en la sistematización de programas dirigidos a la formación de los ciudadanos en el uso efectivo y aprovechamiento de las TIC, como un medio útil y fundamental para su efectiva participación en asuntos de interés colectivo, así como el poco fortalecimiento de proyectos para la construcción y funcionamiento de redes que viabilicen el flujo de información entre los diferentes actores sociales.

Ante esta realidad, nos proponemos como objetivo central de este trabajo analizar el aprovechamiento de las TIC en mecanismos de participación ciudadana en el ámbito local, con el propósito de ofrecer lineamientos generales que orienten la incorporación de éstas como una herramienta útil para el proceso participativo.

Para tal fin, hemos seleccionado cuatro componentes donde las tecnolo- gías constituyen un elemento esencial para apoyar los mecanismos de participación, a saber: unidades de información automatizada, redes basadas en tecnologías de información, Gobierno electrónico (e-goverment) y capacitación tecnológica; lo cuales fueron objeto de una revisión teórica, para extraer las dimensiones fundamentales que determinaran la construcción de lineamientos útiles en el desarrollo de la participación ciudadana.

\section{Planteamientos teóricos-metodológicos.}

En la revisión teórica realizada sobre las TIC, se encontró una mezcla de posturas filosóficas, antecedentes históricos, explicación de problemas, modelos de funcionamiento y acciones de instrumentación, lo cual sustentó la selección de las opciones donde éstas intervienen para potenciar las ventajas de la participación dentro de los contextos locales. Tales componentes se describen considerando su definición teórica, lineamientos metodológicos para su instrumentación y bondades orientadas al beneficio de los actores que hacen vida en las localidades, a saber:

- Unidades de Información Automatizada

La llamada Sociedad de la Información, se concreta en la medida que existan infraestructuras de información integradas por componentes físicos y tecnológicos que permitan ejecutar los procesos que conllevan a acceder a los contenidos que se ofrecen, por diversos tipos de usuarios. En el ámbito de la administración pública, tales infraestructuras se 
encuentran conformadas por unidades, sistemas, servicios y redes, cuya misión radica en ofrecer la oportunidad a los funcionarios públicos de obtener información útil para regular su actividad laboral y coordinar programas, proyectos y acciones entre organismos, ámbitos y niveles jerárquicos. Del mismo modo, facilitan información pública y de cultura general a los ciudadanos, con el fin de que puedan conocer sus derechos, cumplir sus obligaciones, realizar trámites-procedimientos y acceder con mayor facilidad a servicios públicos, entre otros.

El nivel mínimo de estas infraestructuras en los entes municipales, viene dado por la presencia de unidades de información, entendidas como: "un lugar donde la gente puede sacar sentido de todos los datos y acelerar su transmisión a modo de información a la persona adecuada, en el lugar indicado y en el momento preciso" (Timmerman, 2000:78). Señala este autor que cuando estas unidades utilizan las TIC como soporte básico se les denominan unidades de información automatizadas, concebidas como "cámaras de compensación de información" cuya verdadera responsabilidad es convertir los datos en información mediante funciones de recogida, clasificación, análisis, integración y empaquetado para ser remitida a los destinos que las ameriten.

Este tipo de unidades constituye un requisito básico para garantizar a todos los sectores comunitarios el acceso a información de interés requerida para participar en los procesos sociales y suelen ser idóneas, en la medida que se encuentren más cercanas al ciudadano y ofrezcan un aprovechamiento más inclu- yente de las TIC mediante diversas modalidades como: oficinas de información, bibliotecas, archivos, e infocentros.

Las alcaldías venezolanas, en la actualidad han optado por la creación de las denominadas salas técnicas de información integral automatizada, en cumplimiento de la Ley de los Consejos Locales de Planificación Pública en su Art. 19 (Asamblea Nacional, 2002), cuyas funciones están llamadas a proveer la información sectorial codificada necesaria para la planificación, el control de gestión y la participación; así como garantizar aquellos contenidos relacionadas con el registro y control de las comunidades organizadas.

La creación y funcionamiento de estas unidades, pueden encontrarse en la ley de los CLPP cuando hace referencia a su estructuración conformada por la Unidad de Información Computarizada, que comprende la Red Informática Municipal interna y la Red Comunicacional con la sociedad civil organizada y cuerpos de Seguridad del Estado.

En cuanto a las oficinas de información, cada ente público establece sus propios criterios y generalmente están destinadas a suministrar información sobre el mismo ente; mientras que para las bibliotecas, archivos e infocentros, es necesario recurrir a organismos rectores, cuya misión fundamental se centra en la formulación de políticas, lineamientos y demás especificaciones técnicas para el suministro de información y el uso de las tecnologías en el ámbito social. Entre estos organismos se pueden mencionar: el Instituto Autónomo Biblioteca Nacional y de Servicios de Bibliotecas, Archivo Nacional, Bibliotecas Públicas Centrales en 
Tecnologías de información en mecanismos de participación ciudadana Peña, Dionnys; Olivar, Cira y Primera, Nelly

cada Estado, Escuelas de Bibliotecología y Archivología del país, el Ministerio Poder Popular para la Ciencia, Tecnología e Innovación, las oficinas de la Fundación para el desarrollo de la ciencia y la tecnología (FUNDACITE), entre otros.

Esta diversidad de unidades irán conformando según Agudo (2000), los sistemas integrados de información pública, dirigidos a tres conjuntos de acciones: a) las relacionadas con el rescate, potenciación e incluso la creación de los servicios llamados tradicionales de información documental, b) las que tienen que ver con la "automatización" de los procesos y servicios documentales de los sistemas mencionados y c) las vinculadas con la infraestructura teleinformática que incorporan todos los servicios a la gran red.

Los detalles estructurales anteriores, se complementan con lo expuesto por Kothmale (2001) al señalar que para maximizar el potencial de las TIC y evitar desigualdades en su uso, estas unidades deben asegurar: a) una participación activa para responder a las necesidades de información dentro de la comunidad, b) enfoques abiertos y pluralistas ante las diferentes combinaciones posibles de tecnologías para la difusión, suministro y acceso a la información, c) acceso a la Internet, para lo cual debe contarse con una adecuada clasificación y promoción de los sitios y productos web, creando enlaces con los principales motores de búsqueda.

Tomando en consideración los planteamientos expuestos, podemos afirmar que los beneficios para la participación que ofrecen las unidades de información basadas en TIC a los gobiernos mu- nicipales y en consecuencia a toda la población, se traducen fundamentalmente en la posibilidad de contar con espacios o "nichos" donde se encuentren múltiples vías de comunicación e interacción sin importar sus condiciones sociales, económicas, entre otras.

- Redes basadas en tecnologías de información

Las redes son entendidas como "sistemas abiertos" mediante los cuales se produce un intercambio dinámico entre sus propios integrantes y con integrantes de otros grupos y organizaciones, posibilitando así la potencialidad de los recursos que poseen (Bacalini et al., 2004:142), y se caracteriza por permitir la interacción de actores en una determinada localidad e impulsar liderazgos que conllevan al desarrollo de las potencialidades de cada comunidad, dejando atrás el aislamiento para dar paso a la concepción que desde nuestro nacimiento formamos parte de redes, las cuales se van ampliando a medida que nos incorporamos a la sociedad.

Desde una perspectiva simple, esta definición supone que el concepto de redes implica las nociones de compartir e intercambiar información y conocimiento independientemente de que exista una infraestructura formal creada para tales fines, solo depende de la voluntad de participar en función de un progreso común y no individual, o en razón de encontrar salidas a los problemas sociales. Queda claro que el fundamento de estas redes viene dado por el hecho de que la participación de los ciudadanos en la gestión pública se incentiva en la medida en que éstos tengan capacidad de organiza- 
ción y rearticulación entre sus diferentes agrupaciones sociales.

Las ventajas para las redes se acrecientan cuando parte de ellas tienen su origen o son potenciadas por las TIC, puesto que son dinamizadas e incluso constituidas por flujos de información electrónicos, extrapolan el ámbito local para articular, compartir e intercambiar informaciones y recursos a nivel regional, nacional e internacional; de allí emergen nuevos estándares de relaciones sociales, económicas y culturales entre individuos y organizaciones. Estas son llamadas socio-técnicas, porque envuelven la constitución de una organización entre agentes sociales estimulada y mediada por instrumentos tecnológicos y lenguaje codificado, representados por el Internet y la tramas de hiperenlaces en la web (Kauchakje et al., 2006).

Indudablemente estas redes generan nuevas formas de aprovechamiento de la tecnología para activar la participación ciudadana, ello en la medida que se creen según Kothmale (2001), vías de acceso a bases de datos que permitan a cada miembro acumular conocimientos sobre las necesidades fundamentales de la comunidad, reduciendo los costos operativos y de producción de contenidos intercambiando información y adaptando programas ya existentes.

Los lineamientos anteriores son reforzados por Kauchakje et al. (2006), al referir que la constante transformación de la arquitectura de los hiperenlaces en la Web, representa un proceso de continua apropiación social de la tecnología, dinamizada por la vida cotidiana local, las vivencias y preocupaciones presentes en los actores de la comunidad y las expectativas de éstos, expresando las intencionalidades de los actores locales en la definición de nuevas relaciones sociales apoyadas en nuevos hiperenlaces.

Así encontramos redes basadas en tecnologías que permiten el intercambio de información, experiencias y saberes, a la vez que constituyen un espacio para el diálogo y la coordinación en función de metas comunes, una alternativa para compartir recursos y propender al ejercicio de una gestión local participativa.

- Gobierno electrónico

El gobierno electrónico, conocido a su vez como e-gobierno o e-goverment, surge como una estrategia basada en el aprovechamiento de las TIC por parte de las administraciones públicas para mejorar la prestación de servicios y el desarrollo de actividades focalizadas en la satisfacción de las necesidades de la sociedad. Teóricamente esta tendencia revela diversas concepciones orientadas hacia la transformación de las tecnologías en un conjunto de servicios para la participación y apoyo a la gestión pública, así como la generación de vías para la interconexión e interacción múltiple potenciadas fundamentalmente por las paginas Web, intranets, extranets, correos electrónicos, videoconferencias, entre otros recursos.

En general, la finalidad del e-goverment se traduce en proveer más y mejor prestación de servicios a los ciudadanos por parte de gestión pública, en el sentido de brindar puntos de acceso unificados y sencillos para satisfacer múltiples necesidades informativas, adicionar productividad, proveer atención "a la medida" se- 
Tecnologías de información en mecanismos de participación ciudadana Peña, Dionnys; Olivar, Cira y Primera, Nelly

gún los requerimientos de cada usuario; resolver trámites, realizar consultas, hacer reclamos o sugerencia "en línea", aumentar la calidad y reducir el costo (en dinero, tiempo, molestia y carga anímica) de las transacciones dentro del Estado (Tesoro et al., 2002:3).

Desde el punto de vista de la gestión local, el e-gobierno busca potenciar conceptos más trascendentales como la comunicación e interacción para legitimar la gobernabilidad en los espacios del municipio, es decir, se da paso a la participación mediante la estimulación de redes comunitarias, uso de portales y sitios web, componentes ilustrativos que funjan como espejos de las bondades informativas que poseen las instituciones municipales. Los beneficios son innumerables, no sólo en tiempo sino en proyección, imagen e intercambio de experiencias de gestión exitosa en el sector público nacional, que pueden ser replicadas desde lo local.

Lo anterior, aunque representa importantes ventajas no significa la apertura de un e-gobierno participativo hasta tanto se consolide como medio para interacción de los ciudadanos con sus representantes y entes gubernamentales. Solo se constituye en estrategia innovadora en el instante que los ciudadanos puedan utilizar sus servicios y realizar trámites que comúnmente requieren presencialidad, tales como: cancelación de servicios públicos, impuestos y multas; adquisición de cartas de residencia y buena conducta, solvencias municipales, acceder a información institucional (planes, informes de gestión, rendición de cuentas), recibir asesoría técnica on line, llenar formularios para censos e inscripciones de posi- bles evento organizado en dichas instituciones, por mencionar algunos.

Las opciones de servicios en los portales son viables mediante el manejo y adaptación de herramientas como: foros, chat, correos electrónicos, formularios on line y otras opciones que emerjan en el mercado. Con ello sin duda se contribuye al impulso de una cultura participativa, donde se despierte el interés por intervenir en los asuntos públicos de manera rápida y eficiente por medio del uso y aprovechamiento de las TIC.

Ahora bien, la creación y puesta en funcionamiento de estos portales en contextos locales requiere de algunas directrices básicas planteadas por Porrúa (2003), Araya y Porrúa (2004:48,49), a saber:

a) Considerar la situación actual de los recursos tecnológicos e informacionales disponibles en la organización, la visión estratégica del tipo de portal que se quiere construir y los objetivos para materializarla, áreas de acción con su nivel de prioridad y respectivas actividades, aspectos institucionales legales y de seguridad a ser incluidos, estrategias de marketing interno y externo, previsión de riesgos y mecanismos para combatirlos y el calendario de acción para iniciar el proyecto, entre las condiciones y elementos previos a su diseño.

b) Definir la fase en que aspiran desarrollar sus portales, ya sea ésta de información basada en un amplia plataforma y presencia en la web; de interacción entre el ciudadano y el gobierno bajo diálogos virtuales y, de transacción para realizar trámites en línea o integración y transformación que induzcan a rediseñar procesos y 
cambiar normas, siendo esta última el punto ideal de llegada.

c) Definir el papel de los distintos actores y su interacción: gobierno, sector privado y sociedad civil; donde el papel del gobierno es regular y establecer estándares, incluyendo marcos legales, liderar o mostrar el camino; autenticar personas, documentos y transacciones; proteger la privacidad y la seguridad (Reilly y Echeberria, 2003:20).

d) Establecer contenidos en función de las necesidades de la población y de la información que se les quiera suministrar, o bien del tipo de trámites que se puedan llevar a cabo en los portales, todo ello, depende a su vez de la concepción de participación que maneje la autoridad pública, del alcance de sus recursos informacionales, comunicacionales y tecnológicos además del valor presupuestal y consciente que se le otorguen.

Finalmente, las condiciones mínimas para implementar este tipo de estrategia desde las instituciones públicas, implica el conocimiento pleno de sus procesos administrativos, a fin de adecuarlos y automatizarlos eficientemente en búsqueda de los mejores costos e inversión, hasta llegar a aspectos más complejos precisados por Orrego (2004) como: existencia de una infraestructura mínima de software, hardware y conectividad; poseer capacidad tecnológica; afianzar o promover un liderazgo claro y fuerte, no sólo a niveles intermedio sino desde la máxima autoridad; diseñar políticas públicas orientadas a fortalecer este ámbito tecnológico en aspectos como la seguridad informática, inviolabilidad de la co- municación, privacidad de la información y desarrollo de proyectos de alto impacto y corto plazo, que permitan la legitimidad y el posicionamiento para masificar el gobierno electrónico.

Además de estos aspectos, resulta fundamental el impulso de una cultura participativa, pro-tecnológica, donde se despierte el interés por intervenir en los asuntos públicos de manera rápida y eficiente por esa vía, haciendo posible el aprovechamiento de los servicios dispuestos para los diferentes tipos de usuarios.

\section{- Capacitación tecnológica}

La necesidad de garantizar un mayor y mejor acceso a las TIC por parte de la población tiene como aliada a la educación, entendiendo ésta figura como el proceso continuo que busca desarrollar en el ser humano, capacidades, aptitudes y actitudes, como preparación para ser motor fundamental en las actividades sociales, económicas y políticas de la sociedad (PNUD, 2002:97). Esta intención se logra materializar mediante la capacitación tecnológica como estrategia clave para facilitar la adecuada interacción de los sujetos con los medios informáticos y telemáticos, logrando que se descubran sus potencialidades para la intervención del entorno.

Ahora bien, la capacitación en materia tecnológica va más allá de enseñar el uso de instrumentos para centrarse en las interacciones participativas que pueden lograrse dentro de la esfera pública y gubernamental ya que según Savater citado por Mazzina (2003) los ciudadanos no están mal formados académicamente sino cívicamente, pues no saben expresar argumentadamente sus demandas sociales, no son capaces de discernir en 
Tecnologías de información en mecanismos de participación ciudadana Peña, Dionnys; Olivar, Cira y Primera, Nelly

un texto sencillo o en un discurso político lo que hay de sustancia cerebral y lo que es mera hojarasca demagógica, desconocen los valores que deben ser compartidos y aquellos contra los que es urgente rebelarse.

Obviamente, en condiciones de pasividad e inconciencia social la enseñanza de las TIC puede convertirse en un arma de doble filo, por lo tanto, la capacitación en este sentido debe dirigirse hacia la democracia y la participación, entendiendo que no solo se concentre en la transmisión de conocimientos o contenidos, sino que propenda a generar conductas sociales responsables en la medida que atienda a principios fundamentales de desarrollo individual y ciudadano, buscando beneficiar al conjunto de la sociedad. Esto pasa por entender que el ser humano alcanza su propio perfeccionamiento al interactuar continuamente con otras personas, siendo las TIC el medio adecuado para tal fin.

En líneas generales, la intención de la capacitación tecnológica es lograr un desarrollo humano significativo, manifestado en las habilidades y destrezas adquiridas para acceder y manejar los instrumentos que le permitan aprovechar las oportunidades de crecimiento que se le presenten. Mientras más instrumentos maneje cada ciudadano con sentido y significado para sus vidas, mayores posibilidades de desarrollo individual tendrá, y por ende su país (PNUD, 2002:112); pues estará en capacidad de integrarse a la gestión pública empleando diferentes vías para masificar opiniones y propuestas hasta lograr ser escuchado, lo cual requiere nuevas competencias.
Generalmente, suele ocurrir que tanto los organismos político-administrativos como la propia ciudadanía, no están preparados para modificar sus dinámicas actuales de trabajo y extender la gestión participativa a todos los ámbitos decisorios municipales. De tal manera, se considera importante iniciar procesos de enseñanza/aprendizaje que viabilicen los canales de participación acudiendo a las siguientes estrategias metodológicas:

1. Experimentar procesos de aprendizaje tanto entre los ciudadanos como entre los políticos y técnicos de la administración que favorezcan la implantación de una cultura política, activa y deliberativa.

2. Permitir la utilización de herramientas tecnológicas simples para distribuir informaciones sobre las actividades participativas de la ciudad y se irán proporcionando funcionalidades más potentes en la medida que el municipio vaya requiriendo de ellas.

3. Aumentar la cobertura y aprendizaje de las TIC mediante el diseño de planes estadales y municipales donde éstas se incorporen como herramientas de uso obligatorio en los niveles de preescolar, básica y media, integrando todos los actores y roles en comunidades virtuales de aprendizaje, para lo que se requiere contar con aplicaciones y contenidos pertinentes a cada uno (PNUD, 2002).

4. Generar polos para la informatización pública de la sociedad, que incorpore a los sectores excluidos de la población en el uso de información para la resolución de problemas: uno de estos polos avanzaría desde los servicios tradicionales, orales, audiovisua- 
les e impresos, hacia la información digital. El otro polo partiría de los servicios teleinformáticos, digitalizados y en línea, hacia la incorporación de tales posibilidades tecnológicas en el seno de los servicios tradicionales para la popularización de los mismos por medio de alianzas del Estado con las empresas de servicios teleinformáticas (Agudo, 2000).

5. Promover la capacitación técnica de quienes a su vez serán formadores de usuarios, mediante programas continuos con la preparación de contenidos y aplicaciones pertinentes, fiables, adaptadas a las características de la comunidad y las necesidades de los usuarios en materia de información (Kothmale, 2001).

El papel del recurso humano que labora en las instituciones públicas municipales resulta preponderante para el desarrollo de las capacidades técnicas en el manejo de las TIC, pues de estos entes partirán los programas de capacitación permanentes dirigidos a la población. A su vez los ciudadanos adiestrados en el arte de participar, deben ser demandantes de estos beneficios de capacitación, sobre todo por las presiones que impone el uso de dichas tecnologías en el seno de los nuevos mecanismos de participación como los consejos comunales.

\section{Lineamientos para el aprovechamiento de las TIC: Que hacer y como hacerlo.}

Una vez analizada la presencia de las TIC en mecanismos de participación ciudadana en el ámbito local, desde una perspectiva teórica-metodológica se hace necesario plantear lineamientos que pudieran gestarse en el ámbito municipal para lograr un efectivo impacto en la usabilidad y utilidad de las tecnologías, tanto a lo interno de la gestión municipal como en los ciudadanos dispuestos a ejercer acciones participativas en sus comunidades.

En este sentido, se dan parámetros para la capacitación de los ciudadanos en el manejo de las tecnologías, como requisito indispensable para hacer un uso eficiente de la información y la participación; la creación de un circuito informacional, integrado por unidades automatizadas, sistemas de información comunitarios y oficinas de atención a los ciudadanos, con miras a la conformación de redes socio-técnicas; y finalmente el desarrollo de portales participativos que faciliten la interacción gobierno local-ciudadanos, los cuales se plantean a continuación:

A. Programa de capacitación tecnológica:

La participación de los ciudadanos/as en la gestión pública, se ha constituido en los últimos años tanto en el discurso oficial como en la planificación gubernamental, en un mecanismo capaz de lograr mejores niveles de eficiencia, eficacia y transparencia en la administración pública, al abrir escenarios donde la sociedad en conjunto con el gobierno intervienen en muchos aspectos de interés colectivo que impactan su calidad de vida; pero esta intervención se hará posible en la medida que los ciudadanos desarrollen capacidades informativas y tecnológicas para activarla y puedan disponer de espacios propicios para el intercambio de experiencias, comentarios y sugerencias viables ajustadas a cada realidad. 
Tecnologías de información en mecanismos de participación ciudadana Peña, Dionnys; Olivar, Cira y Primera, Nelly

Desde este escenario, surge nuestro interés por la ejecución de programas de capacitación orientados a desarrollar o consolidar en los ciudadanos, conocimientos sobre herramientas tecnológicas, valorización de la información y el conocimiento para la acción, a través de contenidos que abordan los principios participativos y cooperativos entre las comunidades y sus organizaciones, en concordancia con los preceptos constitucionales; la incorporación, uso, aplicación y aprovechamiento de las TIC y la promulgación de los valores de solidaridad, compromiso, apoyo mutuo, los cuales identifican mecanismos de participación como: los consejos locales de planificación pública y los consejos comunales.

El curso comprende cuatro fases, que van desde las actividades previas hasta la fase de cierre con las actividades administrativas inherentes a la consolidación del mismo (véase Cuadro 1) y está integrado por módulos, cuyo contenido está referido a: terminología básica, mecanismos de participación según la normativa vigente, información en el ámbito local, usabilidad y utilidad de las tecnologías de información el contexto local. Estos módulos pueden aplicarse independientemente, según la elección de los representantes comunales o en su totalidad y presentan una interrelación que permite aplicar el enfoque sistémico, donde cada contenido puede integrarse al siguiente.

Una selección de estrategias instruccionales idóneas para acercarse al ciudadano y facilitarle el intercambio de conocimientos y experiencias, de modo que se produzca una relación activa entre los ciudadanos y los facilitadores, visualizan el éxito de la capacitación. Entre ellas se destacan: juegos y dinámicas, discusiones grupales, exposiciones interactivas, ejercicios, simulaciones, asesorias y conversatorios. Los recursos necesarios para la aplicación de estas estrategias se establecerán en los convenios descritos en la segunda fase.

Para la evaluación de los resultados se procederá a aplicar sondeos permanentes para ir evidenciando el aprendizaje significativo obtenido por los ciudadanos, y se les otorgará certificación a aquellos que demuestren la adquisición de las competencias requeridas, lo cual podrá acreditarlos como facilitadores en sus respectivas comunidades, para poder extender la capacitación a todos los ciudadanos.

\section{B. Circuito informacional para}

\section{los municipios:}

La conjugación de los elementos información-tecnologías en los municipios, puede dar origen a una variedad de servicios, tales como unidades de información automatizadas, sistemas de información comunitaria, oficinas de atención a los ciudadanos y redes, que integrados bajo una misma coordinación constituyen un circuito informacional, propuesta que a continuación se presenta de manera muy generalizada.

Unidad de Información Automatizada (UIA)

Unidad compuesta por bases de datos, entendidas éstas como un conjunto o colección de datos interconectados, previamente seleccionados, organizados y almacenados que se encuentran accesibles en tiempo real para cualquier persona, sin distingo de ninguna índole, las cuales serán desarrolladas y gestionadas por especialistas en informática, planificación e información desde las alcaldías. 


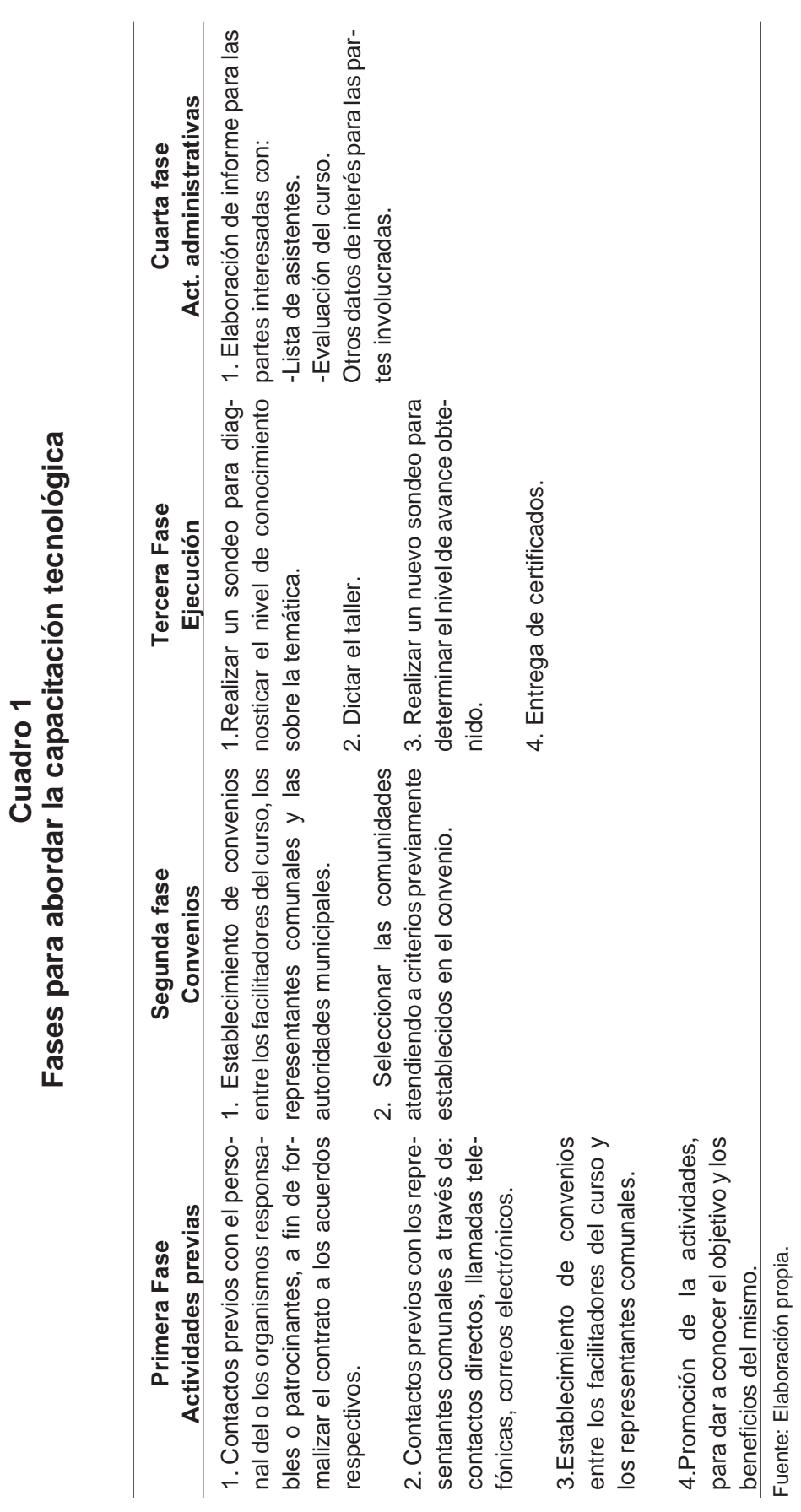


Tecnologías de información en mecanismos de participación ciudadana

Peña, Dionnys; Olivar, Cira y Primera, Nelly

En este sentido, las bases de datos de las UIA facilitarán la correcta realización de los procedimientos informacionales en el municipio, contribuyendo a favorecer la diseminación del caudal de información útil que en él se genera, al suministrarla de manera oportuna a los actores sociales y así apoyar las diversas ocupaciones que éstos desempeñan en sus respectivas comunidades.

Su construcción requiere: a) identificación y selección de fuentes de información; b) búsqueda y revisión del insumo informacional; c) revisión teórica sobre el diseño de base de datos para sustentar los conocimientos básicos de creación y diseño; d) selección y clasificación de la información aplicando criterios de vigencia, pertinencia, relevancia, a través de la lectura y aplicación de un análisis de contenido; e) desarrollo de instrumentos descriptivos según criterios establecidos y necesidades de información expresadas.

La UIA estará ubicada en la estructura organizativa de las alcaldías cumpliendo las siguientes funciones:

- Garantizar información sobre registro y control de las comunidades organizadas, el mapa de necesidades, censo social, directorio de recursos y potencialidades, programas y proyectos, para permitir el seguimiento a las actividades programadas por las alcaldías.

- Asistir técnicamente a las comunidades y Consejos Comunales en el diseño de diagnósticos participativos (recopilar y procesar las propuestas), elaboración de metodologías para formular y presentar los proyectos, evaluación y factibilidad de éstos, así como garantizar la información necesaria para la elaboración del Plan del Presupuesto Anual de Ingresos y Gastos.

- Velar por la coordinación técnica entre los planes.

- Mantener actualizada la información municipal.

- Generar productos informacionales tales como: informes, reportes, estadísticas, que sirvan como medios de consulta a los interesados.

- Formar parte de una red de información municipal de apoyo a las redes sociales.

Además de estas funciones, la UIA se encargará de coordinar acciones con los Sistemas de Información Comunitaria existentes y alimentará la Oficina de Atención al Ciudadano (ver Gráfico 1).

Sistemas de información comunitaria (SIC)

EI SIC se concibe como el conjunto de componentes (personas, hardware, software y datos) que interactúan entre si para apoyar a los Consejos Comunales con información cualitativa que pueda serles útil en el cumplimiento de una gestión exitosa, resaltando en ella la toma de decisiones acertadas, la evaluación de la gestión y la transparencia en las acciones comunitarias que se corresponden con las funciones propias de estos entes.

EI SIC podría instalarse en organismos públicos, privados u ONGs insertos en las comunidades donde funcione el consejo comunal promotor. Sus funciones radican en el aprovechamiento de las TIC para: a) mantener informados a sus usuarios sobre lo que ocurre en su comunidad específica, en cuanto a: planes y 


\section{Gráfico 1 \\ Circuito informacional para los municipios}

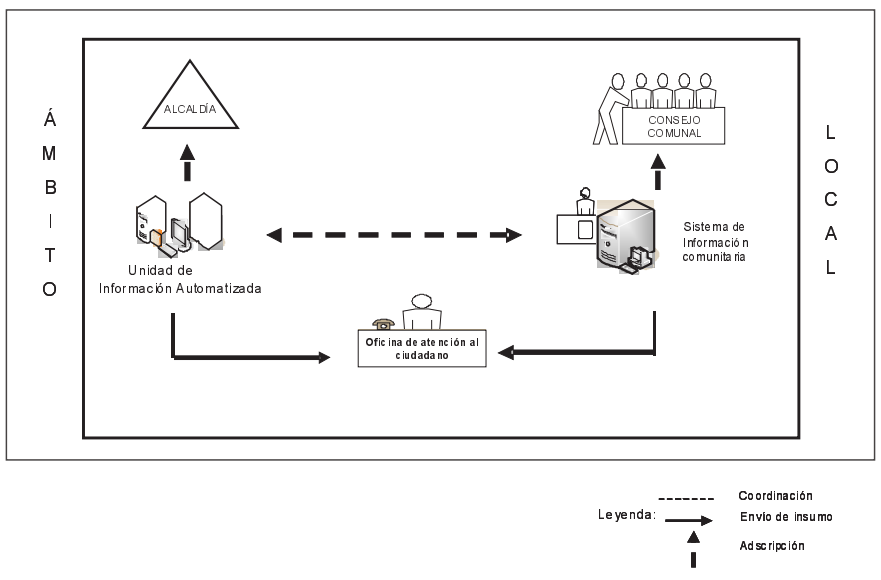

Fuente: Elaboración propia.

proyectos, censo social y demográfico, desempeño de los consejos comunales y de cada de uno de sus componentes estructurales; b) actualizar toda la data o información periódicamente para asignarle veracidad y vigencia, c) atender de manera oportuna las demandas informacionales relacionadas con: solución de problemas, aprovechamiento de oportunidades, identificación de amenazas, diseño de políticas, estrategias y normativas.

Las salidas de un SIC estarán conformadas por productos y servicios, entre los cuales destacan:

a) Recopilación de documentos jurídicos que soportan la participación ciudadana, por ejemplo leyes, decretos, ordenanzas y otros).

b) Informes, reportes del personal encargado de cada una de las dependencias de los consejos comunales (a quién acudir); del municipio (reseña histórica, guías de actividades recreativas, culturales, entre otros); de la gestión de los Consejos Comunales (soluciones a las comunidades, proyectos en marcha, seguimiento de los proyectos).

c) Información sobre actividades de capacitación y adiestramiento (instituciones, cursos, talleres, y otros).

d) Material bibliográfico sobre tendencias en participación, información, tecnologías, gestión local, otros.

e) Estadísticas sobre diferentes aspectos.

EI SIC trabajará en estrecha relación con la UIA para el intercambio de información y asesoramiento técnico en cuanto a la actualización de las tecnologías y en la generación y difusión de los productos informacionales (Ver Gráfico 1). Simultáneamente, podrán actuar bajo la figura de alianzas y convenios con otros SIC para lograr eficiencia, mediante el aprovechamiento de los recursos disponibles cuando existan varios consejos comunales en sectores cercanos. 
Tecnologías de información en mecanismos de participación ciudadana Peña, Dionnys; Olivar, Cira y Primera, Nelly

Oficina Atención al Ciudadano (OAC)

Es una oficina de servicio de cara al ciudadano, destinada a la atención de sus necesidades o demandas puntuales. Su función fundamental estará centrada en el suministro de la información previamente procesada por la UIA y por el SIC (Ver Gráfico 1), y en el apoyo a la formación de las comunidades. La responsabilidad de esta oficina recae sobre los Consejos Comunales y su operatividad pudiera estar en manos de los ciudadanos previamente capacitados, estudiantes becados por las alcaldías, o asignados a través de convenios con las universidades, en cumplimiento a la Ley de Servicio Comunitario del Estudiante de Educación Superior (2005), (Asamblea Nacional, 2005).

Cada OAC estará ubicada en la comunidad a la cual corresponda el CC y dispondrá de una infraestuctura física mínima, constituida por un cubículo o módulo de atención con su equipamiento tecnológico (teléfono, computador con conexión a internet) necesarios para el procesamiento de las solicitudes ante la UIA y el SIC.

Esta oficina trabajará sobre la base de valores inherentes al ser humano como integrante de una comunidad, entre ellos: compromiso social, cooperación, solidaridad, empatía, respeto y responsabilidad en el otorgamiento de servicios, asociados a las necesidades informativas de los ciudadanos, tales como: noticias generales sobre capacitación, eventos y beneficios; avances de las comunidades (proyectos en marcha, aprobaciones); informes de gestión de sus representantes gubernamentales y locales; orientaciones sobre trámites y procedimientos; difusión de campañas en diferentes campos, entre otros planteamientos e inquietudes particulares formuladas.

Redes basadas en tecnologías

Cabe destacar que tanto los SIC y las OAC pueden conformar redes constituidas por flujos de información electrónica, que se fortalecerán cuando coexistan en los municipios, otras modalidades de unidades de información basadas en tecnologías, tales como bibliotecas comunales, salas de lectura, archivos e infocentros.

La ampliación de las redes en el ámbito municipal, abrirá más espacios de diálogo, mayor interacción de los actores en cada una de las comunidades, desarrollo del sentido de pertenencia y capacidad de organización en la población, potencial uso de los recursos, apropiación social de las tecnologías, por citar algunos de los beneficios que pueden ser aprovechados por los ciudadanos, independientemente de su posición social y de la labor que desempeña en su comunidad.

Las redes ya fortalecidas pueden extrapolar el ámbito local para articular recursos info-tecnológicos con otras redes a nivel regional, nacional e incluso internacional, emergiendo así nuevos modelos de relaciones entre ciudadanos y organizaciones.

\section{Portales participativos:}

La incorporación y asimilación de las TIC como instrumentos de participación figura como uno de los más importantes retos de la gestión pública local para insertarse en una sociedad de información igualitaria. Una de las vías más aptas para lograr estos fines, es a través de la creación o rediseño de portales participativos administrados desde las gobernaciones y que permitan a las alcaldías incorporar y acceder a la información 
inherente a los municipios que representan, entendiendo que estos portales constituyen un punto de entrada en Internet donde cualquier usuario puede obtener información pública, previamente organizada y actualizada sobre servicios, trámites y procedimientos, gestión de las autoridades y otras de interés, ello, con la finalidad de facilitar la interacción gobierno local/comunidad.

Para la elaboración de un portal es necesario definir los servicios factibles a ser ofrecidos, lo cual se puede trabajar en dos bloques paralelos de programación: a) el generador de contenidos y b) las aplicaciones y servicios del portal, enfocando como elementos clave la sencillez en la navegación y la capacidad para futuras expansiones del sistema. Operacionalizar las actividades implica comenzar con la arquitectura de contenidos del portal y establecer un diseño gráfico, orientado hacia la practicidad de un portal modular con manejo de información centralizada, con bases de datos distribuidas y esquemas efectivos de seguridad, todo ello acorde con la iconografía y tipografía del municipio correspondiente.

De igual modo, se requiere configurar los módulos de programación estándares y personalizados, describiendo la funcionalidad de los mismos y considerar un análisis de la plataforma a utilizar para el desarrollo del portal, lo cual incluye elementos como el tipo de sistema operativo, software de servidor (http o servidor web), lenguaje de programación o herramienta generadora de portales, y manejador de base de datos. Este análisis permite elegir un generador de portales que posibilite la creación de páginas web dinámicas y multiplataforma con propieda- des como: código fuente abierta que se pueda mejorar y adaptar, ejecución directa en el servidor, de libre distribución y que maneje diversidad de bases de datos, entre otras ventajas de flexibililidad.

Otros criterios a tomar en cuenta para la elección de esta plataforma son los siguientes: universalidad de la aplicación desde el punto de vista de su operatividad y mantenimiento; desarrollo modular e independiente, de rápida actualización por parte de personal no involucrado en su construcción; y transparencia en las nuevas versiones sin afectar los desarrollos realizados

El aprovechamiento de esta tecnología se encuentra centrado en la posibilidad que ofrece de representar la dinámica global de la gestión local en formato digital, con contenidos referidos a los aspectos: geográfico (estado en el cual se encuentra inmerso el municipio), político (gobierno estatal), social (mecanismos de participación vigentes, servicios públicos), tecnológico (sitios de contacto, trámites y procedimientos on line, foros de discusión, chats, enlaces, suscriptores, preguntas frecuentes y descargas), entre otros.

Con estas posibilidades, el ciudadano podrá ejercer un mayor control de su vida y de las actividades que realiza, y por su parte, las autoridades gubernamentales canalizarán una gestión eficiente que impacte en el bienestar de las comunidades.

\section{Conclusiones}

Sin duda alguna, el uso de las TIC en las administraciones públicas es un reto que acerca a las sociedades a un nuevo horizonte sin fronteras de información, conocimiento e intercambio de ex- 
Tecnologías de información en mecanismos de participación ciudadana

Peña, Dionnys; Olivar, Cira y Primera, Nelly

periencias en el siglo XXI. Asimismo, perfilan la nueva gestión pública basada en los principios de transparencia, control social y rendición de cuentas, apoyada en las bondades que éstas ofrecen para la cooperación, la colaboración y la interacción en contextos locales.

Así se concluye que las TIC son aprovechadas mediante la incorporación de componentes clave, tales como: las unidades de información automatizadas, redes basadas en tecnologías y gobierno electrónico, pero ello requiere el desarrollo de capacidades y habilidades para la interacción con los medios informáticos-telemáticos, su utilización y evaluación, para poder descubrir potencialidades y ventajas de éstas para la intervención en su entorno.

Los componentes mencionados conforman infraestructuras utilizadas para a) convertir datos en información mediante labores de recogida, clasificación, análisis, integración y difusión de este insumo, de tal forma que sea posible accederlo de manera expedita para los requerimientos de los funcionarios públicos y los ciudadanos en el contexto local; b) el aprovechamiento de los recursos que ofrece la Internet y la formación de tramas de hiperenlaces para formar lazos de interrelación entre emisores y receptores (nodos), que se hagan efectivos para la cooperación y coordinación social, política, económica y cultural de los municipios; c) la generación de vías de interacción e interconexión múltiple potenciadas por los recursos de la telemática, lo cual provee más y mejores prestaciones a la gestión pública y a los ciudadanos, en el sentido de brindar puntos de acceso unificados y realizar innumerables transacciones.
Los hallazgos teórico-metodológicos anteriores conllevaron a la generación de lineamientos puntuales, factibles de ser aplicables en la realidad venezolana para el aprovechamiento de las TIC en mecanismos de participación como los CLPP y CC. Tales propuestas comprenden:

A. Parámetros para la capacitación de los ciudadanos en el manejo de las tecnologías, requisito indispensable para hacer un uso eficiente de la información y la participación.

B. Creación de un circuito informacional, integrado por unidades automatizadas, sistemas comunitarios, oficinas de atención a los ciudadanos, desarrollo de portales participativos y conformación de redes socio-técnicas que faciliten la interacción gobierno local-ciudadanos así como también una estrecha vinculación entre los entes gubernamentales, las instituciones sociales y comunidades organizadas.

El desarrollo de estos lineamientos, lleva implícito una serie de acciones que comprenden el compromiso de las alcaldías, cámaras municipales, los propios CLPP y CC, en el sentido de destinar partidas presupuestarias o la búsqueda de recursos mediante proyectos especiales para tales fines, entendiendo que éstos dependerá de las características de cada municipio, tales como: cantidad de la población, existencia de profesionales especializados, espacios físicos existentes. Solo de esta manera se podrán consolidar los circuitos de información en cada municipio del país.

En la medida que se reflexione sobre las posibilidades planteadas y se desarrollen las estrategias o lineamientos 
propuestos, se podrá lograr la efectividad de las TIC en su función comunitaria y potenciar las relaciones de los entes municipales con los ciudadanos, por cuanto se habrá alcanzado: un ciudadano inteligente y preparado para la apropiación y uso de las TIC, organización técnica de la información comunitaria y de los medios para accederla, vías expeditas de comunicación on line y organizaciones sociales interrelacionadas que propendan a desarrollos locales significativos.

\section{Referencias Bibliográficas}

Agudo, Álvaro (2000) Ética en la sociedad de información: Reflexiones desde América Latina y el Caribe. Disponible en: http://webworl.unesco.org/infoethics2000/documents/papers_ agudo.rtf. Consultado: 23/09/06

Araya Dujisin, Rodrigo y Porrúa Vigón, Miguel (2004). América Latina Puntogob. Casos y Tendencias en Gobierno Electrónico. Organización de los Estados Americanos AICD. FLACSO. Chile.

Asamblea Nacional de la República Bolivariana de Venezuela (2002). Ley de los Consejos Locales de Planificación Pública. Gaceta Oficial № 37.463. Venezuela

Asamblea Nacional de la República Bolivariana de Venezuela (2005). Ley del Servicio Comunitario del Estudiante de Educación Superior. Gaceta Oficial № 38.272. Venezuela.

Bacalini, G., Ferraris, S. y Marano, G. (2004). "Centros Educativos para la producción total”. En: Redes. El lenguaje de los vínculos. Dabas, E. y Najmanovich, D. Compiladores. Paidós, Argentina.

Berrizbeitia, Jorge (2003). Tecnologías de Información y el desarrollo de las Comuni- dades. Presidente del Centro Nacional de Tecnologías (CNTI). Venezuela.

De Gregorio, M.; Suárez, L.; Meza, A. y otros (2005). Carta Pública. Caracas, Venezuela.

Eglander, Dafner y Salazar, Consuelo (2005). Creando redes. Cartilla educativa. Boletín № 26. Junio.

Kauchakje, S. Penna, M. Frey, K. y Duarte, F. (2006). Redes socio-técnicas y participación ciudadana: propuestas conceptuales y analíticas para el uso de las TICs. REDES- Revista hispana para el análisis de redes sociales, Vol.11, № 3, Diciembre 2006. Disponible en: http://revista-redes.rediris.es. Consultado: 25/07/07

Kothmale, Sri Lanka (2001). Seminario internacional sobre integración de tecnologías de información y comunicación modernas y tradicionales para el desarrollo de la comunidad. Disponible en: http://www.unesco.org/webworld/ public domain/ kothmale_docs/recommendations_sp.rtf. Consultado: 23/09/06

Mazzina, Constanza (2003). La importancia de la Formación Ciudadana (2003) Disponible en: http://www.iigov.org/dhial/ $? p=40 \_07$. Consultado 26/04/04

Orrego, Larrain (2004). Los caminos hacia el E-Gobierno: estrategias y recomendaciones. En: América Latina Puntogob: Casos y tendencias en Gobierno-e. Editores: Rodrigo Araya Dujisin y Miguel A Porrúa Vigón, Coedición FLACSO Chile/AICD-OEA, Santiago de Chile, pp. 41-54.

Páez, A.; Iribarren, C. y Neuman, M. (2004). Gobierno Electrónico y Administración Pública Local. Revista Razón y Palabra. № 35.

Porrúa Vigón, Miguel A. (2003). Elementos para la creación de una estrategia de Gobierno Electrónico. VIII Congreso 
Tecnologías de información en mecanismos de participación ciudadana

Peña, Dionnys; Olivar, Cira y Primera, Nelly

Internacional del CLAD sobre la Reforma del Estado y de la Administración Publica. Panamá, 28/31 de Octubre.

Programa de Naciones Unidas para el Desarrollo-PNUD (2002). Informe sobre Desarrollo Humano en Venezuela. Intenso Offset. Venezuela.

Reilly Catherine y Echeberria Raúl (2003). El papel del ciudadano y de las OSC en el e-gobierno. Un estudio para América Latina y el Caribe.
Tesoro, José Luis, Arambarri, Alejandro José y González Cao, Rodrigo Luis (2002). Gobierno Electrónico: factores asociados a su desempeño. Hallazgos emergentes de un análisis exploratorio de experiencias nacionales. Instituto Nacional de la Administración Pública. INAP. Dirección de Estudios e Información. Disponible en: http:// www.sgp.gov.ar/inap/publicaciones/docs/sociedad/gobel.pdf

Timmerman, Frederick (2000). Cómo ganar el desafío de la información. Ediciones Deusto. Barcelona, España. 